\title{
RELACIÓN DEL ÍNDICE CINTURA-ESTATURA (ICE) CON CIRCUNFERENCIA CINTURA E ÍNDICE DE CINTURA CADERA COMO PREDICTOR PARA OBESIDAD Y RIESGO METABÓLICO EN ADOLESCENTES DE SECUNDARIA.
}

RELATIONSHIP OF WAIST-HEIGHT RATIO (WHTR) WITH WAIST AND WAIST-HIP RATIO AS A PREDICTOR FOR OBESITY AND METABOLIC RISK IN MIDDLE SCHOOL ADOLESCENTS.

Zermeño-Ugalde Pablo ${ }^{1}$, Gallegos-García Verónica ${ }^{1}$, Castro Ramírez Raúl Arcadio², Gaytán-Hernández Darío ${ }^{1}$.

1 Universidad Autónoma de San Luis Potosí (UASLP), México. 2 Instituto Tecnológico y de Estudios Superiores de Monterrey (ITESM), México.

Citation: Zermeño-Ugalde P., Gallegos-García V., Castro Ramírez R.A., Gaytán-Hernández D., (2020 Relación del índice cintura-talla (ICT) con cintura e Índice de Cintura Cadera como predictor para obesidad y riesgo metabólico en adolescentes de secundaria". Revista Salud Pública y Nutrición, 19 (3), 19-27.

Editor: Esteban G. Ramos Peña, Dr. CS., Universidad Autónoma de Nuevo León, Facultad de Salud Pública y Nutrición, Monterrey Nuevo León, México. Copyright: (C)2020 Zermeño-Ugalde P., et al. This is an open-access article distributed under the terms of Creative Commons Attribution License [CC BY 4.0], which permits unrestricted use, distribution, and reproduction in any medium, provided the original author and source are credited.

Competing interests: The authors have declared that no competing interests exist.

DOI: https://doi.org/10.29105/respyn19.3-3

Recibido: 29 de julio 2020;

Aceptado: 12 de septiembre 2020

Email: pablo.zermeno@uaslp.mx 


\title{
RELACIÓN DEL ÍNDICE CINTURA-ESTATURA (ICE) CON CIRCUNFERENCIA CINTURA E ÍNDICE DE CINTURA CADERA COMO PREDICTOR PARA OBESIDAD Y RIESGO METABÓLICO EN ADOLESCENTES DE SECUNDARIA.
}

\author{
Zermeño-Ugalde Pablo', Gallegos-García Verónicaํㅜ, Castro Ramírez Raúl Arcadio², Gaytán-Hernández Darío \\ 1 Universidad Autónoma de San Luis Potosí (UASLP), México. 2 Instituto Tecnológico y de Estudios Superiores de \\ Monterrey (ITESM), México.
}

\section{RESUMEN}

Introducción. El sobrepeso y obesidad en adolescentes son un problema nutricional, el aumento en la prevalencia es preocupante por los efectos adversos del exceso del peso en salud. El Índice Cintura-Estatura (ICE) $\geq 0.5$ es aceptado como un punto de corte universal y puede ser utilizado para predecir obesidad abdominal/riesgo cardiovascular. Objetivo: Identificar el riesgo de obesidad/riesgo metabólico mediante ICE comparando las medidas antropométricas circunferencia cintura (CC.) e Índice de Cintura Cadera (ICC.) en adolescentes. Material y Método: Se estudiaron 378 adolescentes de 11-17 años, se diagnosticó obesidad abdominal con ICE $\geq 0.5$. Se determinó sensibilidad, especificidad, valor predictivo positivo y negativo, área bajo la curva e intervalo de confianza con base a ICE, CC. e ICC para detectar obesidad, comparándolos y determinar mejor validez para diagnostico en adolescentes. Resultados: EI ICE $\geq 0.5$ se observó en 122 (32.7\%) adolescentes con un promedio de $0.47 \pm 0.07$, siendo el predominio en género masculino con $63(51.6 \%)$, difiriendo del género femenino con 59 (48.4\%). Presentando ICE una sensibilidad del $95 \%$ y especificidad $77 \%$. Conclusiones: EI ICE $\geq 0.5$ es una medida sensible para identificar obesidad en adolescentes y encontrar relación con riesgo metabólico pudiendo ser utilizada de diagnóstico en adolescentes.

Palabras Clave: Índice Cintura-Estatura, Adolescentes, Obesidad.

\section{ABSTRACT}

Introduction: Overweight and obesity in adolescents are a nutritional problem, the increase in the combined prevalence is worrying due to the adverse effects of excess weight on health. The waist-height ratio (WHtR) $\geq 0.5$ is accepted as a universal cut-off point and can be used to predict abdominal obesity/cardiovascular risk. Objective: Identify the risk of obesity/metabolic risk through WHtR comparing anthropometric measures waist circumference (WC.) and waist-hip ratio (WHR). In adolescents. Material and method: 378 adolescents aged 11-17 years were studied, abdominal obesity was diagnosed with a WHtR $\geq 0.5$. Sensitivity, specificity, positive and negative predictive value, area under the curve and confidence interval were determined based on WHtR, WC. and WHR. to detect obesity, comparing them and determining better validity for diagnosis in adolescents. Results: The WHtR $\geq 0.5$ was observed in $122(32.7 \%)$ adolescents, with an average of $0.47 \pm 0.07$, the predominance in the masculine gender being $63(51.6 \%)$, differing from females with 59 (48.4\%). Presenting in WHtR a sensitivity of $95 \%$ and specificity $77 \%$. Conclusions: The WHtR $\geq 0.5$ is a sensitive measure to identify obesity in adolescents and find a relationship with metabolic risk and can be used as a diagnosis in adolescents.

Key words: Waist-height ratio, Adolescents, Obesity. 


\section{Introducción}

En México el sobrepeso y obesidad es un problema nutricional en la población adolescente, el aumento en la prevalencia combinada es preocupante, por los efectos adversos del exceso de peso en la salud. La prevalencia de hombres y mujeres adolescentes (12 a 19 años) según la ENSANUT 2018 (Encuesta Nacional de Salud y Nutrición) de sobrepeso es de $23.8 \%$ y de obesidad $14.6 \%$ con total del $38.4 \%$. Siendo mayor en las mujeres en el total del sobrepeso con obesidad con un $41.1 \%$ a diferencia de los hombres con un $35.8 \%$, con una prevalencia en San Luis Potosí del $16.7 \%$ en obesidad (Encuesta Nacional de Salud y Nutrición, 2018). En la encuestan anterior, la ENSANUT 2016 se obtuvo una prevalencia de sobrepeso con un $22.4 \%$ y de obesidad de $13.9 \%$ (Morales, 2016) con un 36.3\% a nivel nacional. Para los adolescentes, el exceso de peso mediante diagnóstico de obesidad y sobrepeso en 2018 es mayor que la encuesta del 2016. Siendo enfermedades que pueden prevenirse y/o desarrollarse desde la infancia, resultando en un problema de salud pública en México. Las mujeres actualmente con base a la ENSANUT 2018 tienen una prevalencia mayor tanto de sobrepeso (27\%) como de obesidad (14.1\%) a diferencia de la ENSANUT del 2016 con un sobrepeso de $26.4 \%$ y obesidad de $12.8 \%$. Los hombres igualmente con base a la ENSANUT 2018 tienen una prevalencia mayor tanto de sobrepeso con un $20.7 \%$ y de obesidad del $15.1 \%$ a diferencia de la ENSANUT del 2016 con sobrepeso de $18.5 \%$ y obesidad del $15 \%$ (Encuesta Nacional de Salud y Nutrición, 2018). Es posible que, de mantenerse la tasa de aumento registrada en los adolescentes (hombres y mujeres) del 2018, en la próxima encuesta, el aumento en las diferencias de prevalencias pueda ser mayor.

Se han descubierto diferentes factores de riesgo causantes de enfermedades, la obesidad es una de ellas (M. Muñoz, 2016). A pesar de la aparente estabilización en el crecimiento de la prevalencia combinada de sobrepeso y obesidad, es una de las enfermedades con más altas tasas en el mundo, por lo que se requiere redoblar esfuerzos en las estrategias de prevención de sobrepeso y obesidad. La obesidad es el exceso de grasa corporal, siendo un problema de salud pública en países en vías de desarrollo, así como en países desarrollados (Williams, 2015). Se han establecido los marcadores para determinar adiposidad como: circunferencia de cintura (CC.), Índice de cintura cadera (ICC.), Índice de Masa Corporal y últimamente el Índice CinturaEstatura (Huamán, 2017), relacionados con enfermedades y mortalidad. A pesar de la urgente preocupación que la obesidad y el sobrepeso han tenido desde 1980, los datos de todos los países indican que la obesidad se ha convertido en una epidemia mundial, desde 1970s, los datos de la National Health and Nutrition Survey (NHANES) revelan un crecimiento en la población adulta y joven independientemente de la raza, etnia, género o edad. El diagnóstico de la obesidad en la niñez y adolescencia se realiza generalmente mediante el Índice de Masa Corporal (IMC) en base a puntos de corte para la edad y el género.

La adolescencia se refiere al periodo de desarrollo entre la infancia y la adultez. La OMS (Organización Mundial de la Salud) define como a los adolescentes a los individuos entre la edad de 10 a 19 años y ellos abarcan cerca del $20 \%$ de la población mundial (Adesina, 2012). La obesidad en la niñez se ha convertido en una epidemia global con una importancia social y de salud en el mundo. Se estima que 4 millones de muertes y 120 millones de discapacidades a nivel mundial son atribuidas a un Índice de Masa Corporal (IMC) elevado (Caballero, 2007). Estudios mencionan que la adolescencia sufre una variedad de problemas de salud especialmente aquellos con inadecuados hábitos alimentarios, como la inadecuada calidad y cantidad de alimentos que comen, siendo de las principales causas de problemas de peso que usualmente pasan desapercibidas ya que la evaluación del peso no se considera una prioridad en la adolescencia como en los niños (Adesina, 2012). La obesidad en la infancia es de especial preocupación dado su impacto en el desarrollo de múltiples condiciones, está incrementando rápidamente y se ha asociado con la dislipidemia, hipertensión y alteraciones de la glucosa (Carter, 2002). Especialmente los niños que tienen sobrepeso son más propensos a padecer obesidad en edades posteriores. De acuerdo a la Organización Mundial de la Salud, 381 millones de niños menores de 20 años tienen sobrepeso u obesidad en el 2016. En las últimas cuatro décadas la prevalencia del exceso de peso en hombres y mujeres adolescentes se ha incrementado substancialmente desde un 4 al $18 \%$ (Power, 1997). Una encuesta realizada en una escuela pública de Estados Unidos 78\% reportaron que su peso no era una preocupación seria de salud, 
aproximadamente un tercio de los encuestados tenían con obesidad, dos tercios tenían sobrepeso (K. A. Muñoz, 1997).

Las escuelas públicas son adecuados lugares para encaminar los hábitos relacionados con el peso además de una manera para concientizar a la población joven (Kriemler, 2011). Algunas escuelas han demostrado, mejorar la dieta, incrementar la actividad física y disminuir la obesidad (Veugelers, 2005). Las intervenciones son especialmente efectivas cuando se incluyen cambios en la educación. Hay esfuerzos encaminados para disminuir la obesidad que incluyen la alimentación, la actividad física y las intervenciones en las escuelas basadas en la población joven (Hoelscher, 2017)

En adolescentes la grasa abdominal o central incrementa el riesgo metabólico (dislipidemia y resistencia a la insulina) y complicaciones cardiovasculares. Los índices predictivos incluyen Índice de Cintura-Estatura, Circunferencia de Cintura e Índice de Cintura Cadera (Sabah, 2014). La circunferencia de cintura tiene alta sensibilidad y especificidad para la medición de la grasa superior, en personas jóvenes. Por lo tanto, es útil para identificar el sobrepeso u obesidad y el riesgo a desarrollar complicaciones metabólicas (Bacopoulou, 2015). Lo mismo sucede con los predictores de Índice de cintura talla y circunferencia de cintura los cuales son mejores predictores. A pesar de que la circunferencia de cintura es un buen marcador para conocer la grasa central, se requieren de tablas para la edad y el género en adolescentes (Eun-Gyong, 2016). El IMC es un buen indicador del exceso de adiposidad, sin embargo, por sí solo no puede distinguir a los individuos con exceso de grasa corporal de aquellos con elevada masa muscular y no puede reflejar la distribución (S Yajnik, 2004). El sobrepeso $(P \geq 85)$ y obesidad $(P \geq 95)$ en niños y adolescentes $(<18$ años) son diagnosticados mediante los percentiles de IMC para la edad de la Organización Mundial de la Salud (Rodea-Montero, 2014). Sin embargo, es debatible si el IMC y/o los percentiles son predictores precisos para el síndrome metabólico en niños y adolescentes, debido a que reflejan el total de la obesidad.

El diagnóstico de sobrepeso u obesidad con base al IMC no diferencia el reflejo de masa muscular y masa grasa. Estudios han mostrado mayor asociación con la grasa intrabdominal, sin embargo, al no considerar la talla, puede clasificar a las personas con la misma circunferencia de cintura siendo alto o bajos de estatura.

Se encuentran varios métodos de valoración de la composición corporal siendo los más precisos, como la resonancia magnética o tomografía computarizada, sin embargo, no son comunes en la práctica debido a su alto costo y a la exposición a la radiación (Kooy, 1993).

Mientras que la distribución de grasa ha sido considerada obesidad aterogénica, se ha puesto atención en métodos que evalúen la obesidad central, como el Índice Cintura-Estatura (ICE) que ha sido propuesto como un buen predictor de distribución de grasa intrabodominal o visceral, riesgo cardiovascular y mortalidad (Ashwell, 2016; Sabah, 2014). El ICE fue por primera vez utilizado en el estudio de Framingham (Kahn, 2005), propuesto para niños y adolescentes, siendo uno de los más comunes actualmente (Kelly, 2013). El ICE es un método fácil de usar, replicar y de bajo costo pudiendo ser realizado en individuos o una población de personas, de ahí viene la importancia en su empleo (Rodríguez, 2018) siendo buen predictor sobre la morbilidad y mortalidad (Ashwell, 2014; Shen, 2017), el cual se ha llegado a recomendar como una medida antropométrica sustituta por su gran capacidad para identificar individuos con riesgos en la salud independientemente del peso corporal (Corrêa, 2019). Con base a ello se sugiere utilizar en Índice de Cintura-Estatura (ICE), estudios han mostrado que el punto de corte de ICE $\geq 0.5$ es aceptado como un punto de corte universal y puede ser utilizado para predecir obesidad abdominal/riesgo cardiovascular en personas mayores de 6 años (Ashwell, 2005; Eun-Gyong, 2016; Taylor R., 2009).

No hay estudios suficientes en población mexicana que reporten la relación entre el Índice CinturaEstatura con medidas antropométricas para la edad en población adolescente mexicana. Por lo tanto, el principal objetivo del estudio es identificar el riesgo de obesidad/riesgo metabólico mediante Índice de Cintura-Estatura comparando las medidas antropométricas Circunferencia de cintura e Índice Cintura Cadera en población adolescente. En México hay pocos estudios que evalúen en la población 
adolescente la asociación del Índice Cintura-Estatura (ICE) con las medidas antropométricas (ICC y CC.) como predictor para obesidad y riesgo metabólico.

\section{Material y Método}

El estudio está conformado por 378 adolescentes de 11-17 años, de una escuela secundaria de San Luis Potosí, participando mediante una convocatoria abierta a través de la escuela. Se realizó un estudio cuantitativo, correlacional, transversal y observacional en estudiantes de ambos géneros, citados en grupos según el salón del grado escolar de $1^{\circ}$ grado, $2^{\circ}$ y $3^{\circ}$ de secundaria. Para el acceso a la escuela se obtuvo mediante la autorización del Director y Subdirector del plantel, solicitándose carta de consentimiento firmada por el padre/tutor y el adolescente a participar. Los criterios de inclusión tomados en cuenta fueron los siguientes: todos los estudiantes cuyos padres firmen y entreguen la carta de consentimiento informado y asistan a la toma de medidas y que cursen $1^{\circ}$ grado, $2^{\circ}$ y $3^{\circ}$ de secundaria. Los criterios de exclusión fueron los siguientes: estudiantes que ya no se encuentren inscritos en el periodo de estudio. Criterios de eliminación, los adolescentes que por alguna razón no tengan las mediciones antropométricas de: Peso, Talla, Circunferencia de Cintura (CC.) y Circunferencia Cadera (CCa.).

Se realizaron las mediciones dentro de un espacio asignado dentro de la Institución, el cual se proporcionó para adaptarse a realizar las medidas antropométricas de manera individual (Peso, Talla, CC. y CCa.), en el que se canalizo a los estudiantes por grupos en el momento del receso para no interrumpir con sus actividades académicas con una duración aproximada de 5 minutos por alumno.

El estudio tuvo una duración de 4 meses en el proceso de recolección de datos antropométricos por Licenciados en Nutrición previamente capacitados y estandarizados en la toma de medidas Peso, Talla, CC., CCa. El peso se obtuvo en lo estudiantes mediante basculas Tanita ${ }^{\circledR}$ Bc-568, sin zapatos en un área adecuada para la toma de medidas antropometricas, asignada por la institución en donde se tuviera comodidad y mantuviera la privacidad de los estudiantes; La talla en bipedestación sin zapatos a través de estadímetros portátiles Seca ${ }^{\circledR} 213$. Con la medición de peso $(\mathrm{kg})$ y talla $(\mathrm{m} 2)$, se obtuvo como resultado el Î́ndice de masa corporal (IMC) para la edad utilizado en población adolescente, calculado por la división del peso entre la talla al cuadrado en donde se graficaron los resultados con los percentiles de la Organización Mundial de la Salud según la edad, para obtener el resultado del diagnóstico nutricional usando los criterios establecidos por la Organización Mundial de la Salud: siendo normopeso: $\leq 84.9$ Percentil, sobrepeso: $\geq 85$ Percentil y obesidad $\geq 95$. La CC. y CCa. se obtuvieron con cintas métricas Lufkin (metálica flexible con precisión de $0.5 \mathrm{~cm}$ ) en bipedestación entre el momento de inhalación y exhalación del paciente, obteniendo el ICC por medio de la división de CC. (cm) y CCa. (cm). Mediante la medición de CC. y de talla se obtuvo el ICE, obtenido mediante la división de la cintura $(\mathrm{cm})$ entre la estatura $(\mathrm{cm})$, siendo $\geq 0.5$ utilizado como punto de corte. el cual el resultado servirá como medida predictora para detectar riesgo cardiovascular.

Análisis estadístico. Se utilizó estadística descriptiva, media, mediana, moda, mínimo, máximo, rango de desviación estándar. Para la evaluación de las variables de las medidas antropométricas ICE comparado con CC., ICC. para el diagnóstico de nutrición de IMC para la edad, se utilizarán las pruebas estadísticas: correlación lineal de Pearson, regresión lineal y Anova de una vía, considerando 95\% de confianza en todos los casos. Se utilizó el software estadístico SPSS (Statistical Product and Service Solutions) Versión 15. Una vez finalizada la toma de medidas antropométricas en toda la población se vacío la información en una base de datos realizada en el programa de Microsoft Excel y se analizó en el programa estadístico SPSS entregando a la institución los resultados individuales y generales de los estudiantes con una plática informativa para generar conciencia del diagnóstico nutricional de los estudiantes. Para la sensibilidad, especificidad y valores predictivos los resultados se registraron en una base de datos en el programa Excel y fueron procesado en Software estadístico R. Mediante el desarrollo de una regresión logística se determinó la sensibilidad, especificidad, valores predictivos para medir la eficiencia del ICE, ICC. y la CC. para el diagnóstico de obesidad. Se establecen como punto de corte un $50 \%$ como corte en la probabilidad que resultaba de la regresión logística. 


\section{Resultados}

Se estudiaron 372 adolescentes entre 11 y 17 años de edad (195 Masculinos y 177 Femeninos), el promedio de edad fue de $13 \pm .9$ años con y sin obesidad/riesgo metabólico mediante el ICE. La distribución de acuerdo al estado nutricional según IMC/edad por percentiles en adolescentes, se encontró en sobrepeso (Percentil $\geq 85$ ) con 46(12.3\%) adolescentes y con obesidad (Percentil $\geq 95) 99$ (26.6\%) dando un total de 145 (38.9\%) adolescentes con sobrepeso y obesidad por medio de los percentiles de la OMS.

El ICE $\geq 0.5$ se observó en 122 (32.7\%) adolescentes un promedio del ICE de $0.47 \pm 0.07$, con predominio en el género masculino con 63(51.6\%) adolescentes, a diferencia que en el género femenino se presentó un ICE $\geq 0.5$ en $59(48.4 \%)$ adolescentes. Según el IMC/edad de sobrepeso y obesidad (percentil $\geq 85$ y $\geq 95$ ) se encontraron un $91 \%$ de los adolescentes clasificados como obesidad/riesgo metabólico mediante el ICE $\geq 0.5$, y el $13.6 \%$ de los que presentaron diagnóstico normal mediante IMC/edad se encontraron con un ICC $\leq 0.49(p<0.01)$. En los adolescentes masculinos con ICE $\geq 0.5$ se encontraron con $\mathrm{CC} \geq 90 \mathrm{~cm}$ el $34 \%$ y $\geq 102 \mathrm{~cm}$ el $9.5 \%$. En las adolescentes femeninas con un ICE $\geq 0.5$ se encontró en CC. $\geq 80 \mathrm{~cm}$ en el $40.6 \% \mathrm{y} \geq 88 \mathrm{~cm}$ en el $20.3 \%$. En los adolescentes masculinos con un ICE $\geq 0.5$ se encontraron con un ICC $\geq 0.95$ el $1.3 \%$. En las adolescentes femeninas con un ICE $\geq 0.5$ se encontraron con un ICC $\geq 0.86$ el $3.2 \%$. ver tabla 1 .
Tabla 1. Índice Cintura-Estatura (ICE) con punto de corte $\geq 0.5$, según género, edad e IMC/edad.

\begin{tabular}{|c|c|c|c|}
\hline Variable & $\begin{array}{c}\text { ICT } \leq 0.49 \\
N(\%)\end{array}$ & $\begin{array}{c}\text { ICT } \geq 0.5 \\
N(\%)\end{array}$ & $\begin{array}{l}\text { Total } \\
\text { N (\%) }\end{array}$ \\
\hline \multicolumn{4}{|l|}{ GÉNERO } \\
\hline Masculino & $132(52.8)$ & 63 (51.6) & $195(52.4)$ \\
\hline Femenino & $118(47.2)$ & $59(48.4)$ & 177 (47.6) \\
\hline \multicolumn{4}{|l|}{ EDAD } \\
\hline 11-14 años & $230(92.0)$ & $110(90.2)$ & 340 (91.4) \\
\hline 15-17 años & $20(8.0)$ & $12(9.8)$ & $32(8.6)$ \\
\hline \multicolumn{4}{|l|}{ IMC/EDAD } \\
\hline $\begin{array}{l}\text { Normopeso } \\
\text { (Percentil } \leq 84.9 \text { ) }\end{array}$ & $216(86.4)$ & $11(9.0)$ & $227(61.0)$ \\
\hline Sobrepeso y & & & \\
\hline $\begin{array}{l}\text { Obesidad* } \\
\text { (Percentil } \geq 85 \text { ) }\end{array}$ & $34(13.6)$ & $111(91.0)$ & $145(38.9 \%)$ \\
\hline \multicolumn{4}{|l|}{ CINTURA ATP III } \\
\hline \multicolumn{4}{|l|}{ Hombres* } \\
\hline$\leq 101.9$ & $132(100)$ & $57(90.5)$ & 189 (96.9) \\
\hline$\geq 102$ & $0(0)$ & $6(9.5)$ & $6(3.1)$ \\
\hline \multicolumn{4}{|l|}{ Mujeres* } \\
\hline$<87.9$ & $118(100)$ & 47 (79.7) & $165(93.2)$ \\
\hline$\geq=88$ & $0(0)$ & $12(20.3)$ & $12(6.8)$ \\
\hline \multicolumn{4}{|l|}{ ICC } \\
\hline \multicolumn{4}{|l|}{ Hombres* } \\
\hline$\leq 0.94$ & $195(52.4)$ & $53(14.2)$ & $248(66)$ \\
\hline$\geq 0.95$ & $2(0.5)$ & $5(1.3)$ & $7(1.8)$ \\
\hline \multicolumn{4}{|l|}{ Mujeres* } \\
\hline$\leq 0.85$ & $114(30.6)$ & $35(9.4)$ & $149(40)$ \\
\hline$\geq 0.86$ & $3(0.8)$ & $12(3.2)$ & $15(4)$ \\
\hline \multicolumn{4}{|l|}{ CINTURA OMS } \\
\hline \multicolumn{4}{|l|}{ Hombres* } \\
\hline$\leq 89.9$ & $132(35)$ & $30(8)$ & $160(43)$ \\
\hline$\geq 90$ & $0(0)$ & $33(8.8)$ & $33(8.8)$ \\
\hline \multicolumn{4}{|l|}{ Mujeres* } \\
\hline$\leq 79.9$ & $118(31)$ & $17(4.5)$ & $135(36)$ \\
\hline$\geq 80$ & 0 & $42(11)$ & $42(11.2)$ \\
\hline
\end{tabular}

El ICE $\geq 0.5$ en nuestra población mostró sensibilidad mayor en la detección de obesidad con un $95 \%$, el ICC en hombres $94 \%$ y en mujeres $95 \%$, en CC. se mostró una sensibilidad en hombres del $97 \%$ y en mujeres $93 \%$. El área bajo la curva para obesidad fue: En ICE de 0.94, ICC femenino 0.76, ICC masculino 
0.78, CC. masculino 0.95 , CC. femenino 0.94. Ver tabla 2.

Tabla 2. Índice Cintura-Estatura (ICE) como prueba diagnóstica para detectar obesidad comparado con ICC y Circunferencia Cintura (CC.), en adolescentes de San Luis Potosí. n=372

\begin{tabular}{|c|c|c|c|c|c|c|c|c|c|}
\hline \multicolumn{3}{|l|}{ Variables } & \multicolumn{3}{|c|}{ P.Corte \%Sens \%Spec } & \multicolumn{2}{|c|}{ VPP VPN } & \multirow{2}{*}{$\begin{array}{c}\text { IC-95\% } \\
0.84-0.91\end{array}$} & \multirow{2}{*}{$\frac{\mathrm{ABC}}{0.94}$} \\
\hline ICT & Total & Punto de corte & 0.5 & $95 \%$ & $77 \%$ & $86 \%$ & $91 \%$ & & \\
\hline \multirow[t]{3}{*}{ ICC } & Masculino & $\operatorname{Reg} \mathrm{L}$ & 0.9 & $94 \%$ & $42 \%$ & $70 \%$ & $83 \%$ & $0.66-0.79$ & 0.78 \\
\hline & & Punto & 95 & $98 \%$ & $13 \%$ & $62 \%$ & $83 \%$ & $0.56-0.7$ & \\
\hline & Femenino & Punto de corte & 0.86 & $95 \%$ & $24 \%$ & $68 \%$ & $73 \%$ & $0.6-0.75$ & 0.76 \\
\hline \multirow[t]{3}{*}{ Cintura } & Masculino & Reg Log & 78.5 & $97 \%$ & $80 \%$ & $88 \%$ & $94 \%$ & $0.85-0.94$ & 0.95 \\
\hline & & Punto de $c$ & 90 & $99 \%$ & $40 \%$ & $71 \%$ & $97 \%$ & $0.69-0.81$ & \\
\hline & & Punto de corte ATP III. & 102 & $100 \%$ & $8 \%$ & $61 \%$ & $100 \%$ & $0.55-0.69$ & \\
\hline \multirow[t]{3}{*}{ Cintura } & Femenino & $\mathrm{Re}$ & 77 & 93 & $80 \%$ & $89 \%$ & $87 \%$ & 0.82 & \\
\hline & & Punto de & 80 & $95 \%$ & $56 \%$ & $79 \%$ & $88 \%$ & $0.74-0.86$ & 0.94 \\
\hline & & Punto de corte ATP III. & 88 & $100 \%$ & $18 \%$ & $67 \%$ & $100 \%$ & $0.62-0.76$ & \\
\hline
\end{tabular}

Fuente: Encuesta, Medición

P. Corte: Punto de corte; \% Sens: Sensibilidad; \%Spec: Especificidad; VPP: valor predictivo positivo; VPN: valor predictivo negativo; IC-95\%: intervalo de confianza; ABC: Área bajo la curva

\section{Discusión.}

Nuestros resultados demuestran que, en adolescentes, un ICE igual o superior a 0.5 , puede ser un indicador útil para identificar obesidad, además por ser un punto de corte que es aplicable para ambos géneros $\mathrm{y}$ todos los grupos de edad, independientemente de la etnia, hace fácilmente su aplicación e interpretación (Vieira, 2018). Al comparar nuestros resultados frente a los del ICC y a la CC., se demostró que ICE tuvo una sensibilidad similar a la variable de CC. con los cortes establecidos por el modelo de Regresión logística. La especificidad es mayor para la variable de CC., aunque la diferencia no fue significativa. Además, los cortes de probabilidad en la literatura (WHO, 2008) que podemos encontrar para CC. del ICC y CC., resultaron con menos asertividad con aquellos encontrados mediante la regresión logística, como el corte de 0.95 ICC tiene un intervalo de confianza casi 10 puntos menor que el 0.90 que se concluyó mediante el algoritmo.

El síndrome metabólico se refiere a la presencia de varios factores de riesgo cardiovascular, como lo es la obesidad, adiposidad visceral, etc. (Huang, 2009), el ICE se relaciona con el síndrome metabólico como le menciona Yang en su estudio (Yang, 2017), reportándose en nuestro estudio un punto de corte para ICE de $\geq 0.50$ con un $95 \%$ de sensibilidad, $77 \%$ de especificidad para el diagnóstico de obesidad siendo buen predictor, similar a los resultados de Arnaiz y col. encontrando una sensibilidad del $72 \%$, especificidad del $70 \%$ (Valle-leal, 2016). RodeaMontero y col mencionan en su estudio una sensibilidad del $69.1 \%$ y una especificidad del
$57.1 \%$, sin embargo, utilizaron un punto del corte de ICE $\geq 0.6$ (Rodea-Montero, 2014).Además se encontró con sobrepeso y obesidad con base a los percentiles de IMC/edad de la OMS a 145(38.9\%) adolescentes, muy similar a los datos de la ENSANUT del 2018 con un 38.4\%(Encuesta Nacional de Salud y Nutrición, 2018) y del 2016 con $36.3 \%$ (Morales, 2016) con una diferencia de $<0.001$ en los percentiles de sobrepeso y obesidad. En nuestro estudio se encontró en hombres con base a la CC., una sensibilidad del $99 \%$ y una especificidad del $40 \%$, en mujeres unas sensibilidad del $95 \%$ y especificidad del $56 \%$ comparado con el estudio de Rodea-Montero el cual encontraron en hombres una sensibilidad menor con un $74.4 \%$, una especificidad del $65.2 \%$ y en mujeres una sensibilidad menor del $55.2 \%$ con una especificidad mayor del $84.2 \%$ (Rodea-Montero, 2014) comprándolo con base a nuestro estudio, la CC. tiene mayor capacidad para detectar la obesidad en la población de adolescentes. El ICE se ha sugerido que es un indicador de obesidad de fácil obtención, siendo su valor relativamente similar en nuestro estudio en la variable de género masculino y femenino como lo menciona en su estudio P.Arniz y col. con una $\mathrm{p}=0.409$ siendo no significativa (Arnaiz, 2014), sin embargo en este estudio no fue el caso en la edad de la adolescencia, debido a que la población más joven (11-14años) mostro $91.4 \%$ en mayoría sobrepeso u obesidad con base al ICE, como lo sugiere Roswall y col. presentándose en las edades más jóvenes. La medición de ICE es un buen indicador de riesgo cardiovascular, sin embargo, debe de haber referencias según la edad (Roswall et al., 2009). Además, se ha buscado la asociación entre ICE, los factores de riesgo y condiciones de salud, nuestra investigación permitió determinar la concordancia entre el ICE mayor a 0.5 para establecer obesidad. Existen estudios que muestran que el ICE y la CC. son un buen indicador de enfermedad cardiovascular (López-González, 2016; Ma, 2016), sin embargo, la Organización Mundial de la salud utiliza la CC. o el IMC para la población en general para definir obesidad y el riesgo metabólico (WHO, 2008). Cossio y col. mostraron que el ICE y la CC. se relacionaban significativamente en niños hispánicos con los niveles insulina, mostrando una mayor influencia en la obesidad abdominal (Cossio, 2009). Por otra parte, es de suma importancia el resaltar que del total de adolescentes en el estudio con un ICE $\geq 0.5,112(30 \%)$ son clasificados con obesidad según 
el IMC, dando una subestimación de adolescentes que cursan con la condición en su composición corporal de sobrepeso u obesidad, además de su importancia a nivel de la salud de los problemas en escolares.

Una limitación del estudio se pudo observar que es una población que se encuentra en su pico de crecimiento pudiendo alterar los resultados por los cambios en talla y reservas corporales de grasa, dependiendo de la edad en los adolescentes, además de poder variar en la ingesta alimentaria por los cambios de las hormonas estimuladoras del apetito y en la actividad física. En la población mexicana son escasos los estudios sobre crecimiento por lo cual se llegan a utilizar las curvas de crecimiento de la OMS (Van Den Broeck, 2009)

\section{Conclusiones}

En este estudio se demostró que con un criterio de ICE $\geq 0.5$ es una medida sensible para identificar obesidad en adolescentes y encontrar relación con riesgo metabólico, a pesar que en nuestro estudio tuvo una menor especificidad se encontró una gran sensibilidad. Es una herramienta que puede ser utilizada de diagnóstico en la población mexicana, por lo tanto, se recomienda realizar investigación acerca de los puntos de corte dependiendo del tipo de población, etnia y la edad. Se puede concluir que el valor de ICE no varía con el género, pero si en las edades dentro de la etapa de la adolescencia (11 a 17 años). Estos resultados apoyan el uso de la medición de ICE como un método para diagnóstico de obesidad que puede ser utilizado para población adolescente para el inicio y seguimiento de la adolescencia, siendo simple y de bajo costo. La temprana prevención y detección de sobrepeso $\mathrm{u}$ obesidad en edades tempranas es de gran importancia, se debe de poner atención en los cambios de mediciones antropométricas para alertar a los padres o escuelas antes de que se inicien los problemas de salud y recordar el mensaje de "mantén la medición de tu CC. menor que la mitad de tu estatura".

\section{Bibliografía}

Adesina, A. F. (2012). Weight status of adolescents in secondary schools in port Harcourt using Body Mass Index (BMI). Italian Journal of Pediatrics,
38(1), 11-15. https://doi.org/10.1186/18247288-38-31

Arnaiz, P. (2014). La razón cintura estatura en escolares no varía con el género, la edad ni la maduración puberal. Rev Med Chile, 142, 574578.

Ashwell, M. (2005). Six reasons why the waist-toheight ratio is a rapid and effective global indicator for health risks of obesity and how its use could simplify the international public health message on obesity. International Journal of Food Sciences and Nutrition, 56(August), 303307.

https://doi.org/10.1080/09637480500195066

Ashwell, M. (2014). Waist-to-Height Ratio Is More Predictive of Years of Life Lost than Body Mass Index. PLoS ONE, 9(9), 1-11. https://doi.org/10.1371/journal.pone.0103483

Ashwell, M. (2016). Waist-to-height ratio as an indicator of ' early health risk' : simpler and more predictive than using a ' matrix ' based on BMI and waist circumference. BMJ Open, 6, 17. https://doi.org/10.1136/bmjopen-2015-010159

Bacopoulou, F. (2015). Waist circumference, waistto-hip ratio and waist-to-height ratio reference percentiles for abdominal obesity among Greek adolescents. BMC Pediatrics, 15(50), 1-9. https://doi.org/10.1186/s12887-015-0366-z

Caballero, B. (2007). The Global Epidemic of Obesity: An Overview. Epidemiol Rev, 29(7), 15. https://doi.org/10.1093/epirev/mxm012

Carter, R. C. (2002). The Impact of Public Schools on Childhood Obesity. Journal of the American Medical Association, 288(17), 2176. https://doi.org/10.1001/jama.288.17.2176JMS1106-2-1

Corrêa, M. M. (2019). The ability of waist-to-height ratio to identify health risk. Revista de Saúde Pública, 53(66), 1-12.

Cossio, S. (2009). How Do Different Indices of Obesity Correlate with Cardiometabolic Disease Risk Factors in Multiethnic Youths? Endocrine 
Practice, 15(5), 403-409. https://doi.org/10.4158/EP08354.OR

Encuesta Nacional de Salud y Nutrición. (2018). Informe de Resultados de la Encuesta Nacional de Salud y Nutrición 2018. Retrieved September 5, 2020, from https://ensanut.insp.mx/encuestas/ensanut2018/i nformes.php $\% 0 \mathrm{~A}$

Eun-Gyong, Y. (2016). Waist-to-height ratio as a screening tool for obesity and cardiometabolic risk. Korean J Pediatr, 59(11), 425-431.

Hoelscher, D. M. (2017). Surveillance Systems to Track and Evaluate Obesity Prevention Efforts. Annual Review of Public Health, 38(1), 187-214. https://doi.org/10.1146/annurev-publhealth031816-044537

Huamán, J. (2017). Índice cintura-estatura como prueba diagnóstica del Síndrome metabólico en adultos de Trujillo. Rev.Med Hered, 28, 13-20.

Huang, P. L. (2009). A comprehensive definition for metabolic syndrome. Disease Models \& Mechanisms, 237, 231-237. https://doi.org/10.1242/dmm.001180

Kahn, H. S. (2005). A population-based comparison of bmi percentiles and waist-to-height ratio for identifying cardiovascular risk in youth. The Journal of Pediatrics, 146, 482-488.

Kelly, A. S. (2013). Severe Obesity in Children and Adolescents : Identification, Associated Health Risks , and Treatment Approaches. AHA Journals, 128, 1689-1712. https://doi.org/10.1161/CIR.0b013e3182a5cfb3

Kooy, K. van der. (1993). Techniques for the measurement of visceral fat: a practical guide. International Journal of Obesity and Related Metabolic Disorders, 17(4), 187-196.

Kriemler, S. (2011). Effect of school-based interventions on physical activity and fitness in children and adolescents: A review of reviews and systematic update. British Journal of Sports Medicine, 45(11), 923-930. https://doi.org/10.1136/bjsports-2011-090186
López-González, D. (2016). Diagnostic performance of waist circumference measurements for predicting cardiometabolic riesk in mexican children. Endocrine Practice, 22(10), 11701176. https://doi.org/10.4158/EP161291.OR

Ma, L. (2016). Waist Circumference is Better Than Other Anthropometric Indices for Predicting Cardiovascular Disease Risk Factors in Chinese Children--a Cross-Sectional Study in Guangzhou. Journal of Atherosclerosis and Trombosis, 22, 320-329.

Morales, P. K. (2016). Encuesta Nacional de Salud y Epidemiología de Medio Camino 2016. Informe final de resultados. Resultados Nacionales, 2016, 1-149. Retrieved from https://www.gob.mx/cms/uploads/attachment/fil e/209093/ENSANUT.pdf

Muñoz, K. A. (1997). Food Intakes of US Children and Adolescents Compared With Recommendations. Pediatrics, 100, 323-329.

Muñoz, M. (2016). El Índice cintura-Talla como predictor del daño cardiovascular. Rrevista Cubana de Alimentación y Nutrición, 26(2), 239251.

Power, C. (1997). Measurement and long-term health risks of child and adolescent fatness. International Journal of Obesity, 21(7), 507-526. https://doi.org/10.1038/sj.ijo.0800454

Rodea-Montero. (2014). Waist-to-Height Ratio Is a Better Anthropometric Index than Waist Circumference and BMI in Predicting Metabolic Syndrome among Obese Mexican Adolescents. International Journal of Endocrinology, 2014(195407), 1-9.

Rodríguez, J. H. (2018). Utilidad del índice cintura / cadera en la detección del riesgo cardiometabólico en individuos sobrepesos y obesos. Revista Cubana de Endocrinología, 29(2), 1-16.

Roswall, J., Bergman, S., Almqvist-tangen, G., Alm, B., Niklasson, A., \& Nierop, A. F. M. (2009). Population-based waist circumference and waist- 
to-height ratio reference values in preschool children, Acta pediatrica 98(10) 1632-1636. https://doi.org/10.1111/j.1651-

2227.2009.01430.x

S Yajnik, C. (2004). Clinical picture The Y-Y paradox. The Lancet, 363(9403), 163.

Sabah, N. (2014). Body mass index and waist / height ratio for prediction of severity of coronary artery disease. BMC Research Notes, 7(1), 1-7. https://doi.org/10.1186/1756-0500-7-246

Shen, S. (2017). Waist-to-height ratio is an effective indicator for comprehensive cardiovascular health. Scientific Reports, 7(1), 1-7. https://doi.org/10.1038/srep43046

Taylor R. (2009). Predictive Ability of Waist-toHeight in Relation to Adiposity in Children Is Not Improved With Age and Sex-Specific Values. Obesity, 19(5), 1062-1068. https://doi.org/10.1038/oby.2010.217

Valle-leal, J. (2016). Índice cintura-estatura como indicador de riesgo metabólico en niños. Revista Chilena de Pediatría, 87(3), 180-185. https://doi.org/10.1016/j.rchipe.2015.10.011

Van Den Broeck, J. . (2009). The World Health Organization child growth standards: expected implications for clinical and epidemiological research. European Journal of Pediatrics, 168, 247-251. https://doi.org/10.1007/s00431-0080796-9

Veugelers, P. J. (2005). Effectiveness of school programs in preventing childhood obesity: A multilevel comparison. American Journal of Public Health, 95(3), 432-435. https://doi.org/10.2105/AJPH.2004.045898

Vieira, S. A. (2018). Waist-to-height ratio index or the prediction of overweight in children. Rev Paul Pediatr, 36(1), 52-58.

WHO. (2008). Waist Circumference and Waist-Hip Ratio Report of a WHO Expert Consultation. (WHO Document Production, Ed.). Geneva, Switzerland.
Williams, E. P. (2015). Overweight and Obesity: Prevalence, Consequences, and Causes of a Growing Public Health Problem. Curr Obes Rep, 4, 363-370. https://doi.org/10.1007/s13679-015$0169-4$

Yang, H. (2017). Waist-to-height ratio is better than body mass index and waist circumference as a screening criterion for metabolic syndrome in Han Chinese adults. Medicine, 96(39), 1-8. 